\title{
Dielectric Measurements on Sol-Gel Derived Titania Films
}

\author{
RIFAT CAPAN ${ }^{1,4}$ and ASIM K. RAY ${ }^{2,3,5}$ \\ 1.-Physics Department, Science Faculty, Balikesir University, 10100 Balikesir, Turkey. \\ 2.-Department of Materials, Queen Mary College, Mile End Road, London E1 4NS, UK. 3.-Present \\ address: Institute of Materials and Manufacturing, Brunel University London, Uxbridge, Middle- \\ sex UB8 3PH, UK. 4.—e-mail: rcapan@balikesir.edu.tr. 5.—e-mail: Asim.Ray@brunel.ac.uk
}

\begin{abstract}
Alternating current (AC) impedance measurements were performed on $37 \mathrm{~nm}$ thick nanostructured sol-gel derived anatase titania films on ultrasonically cleaned (100) $p$-silicon substrates at temperatures $T$ ranging from $100 \mathrm{~K}$ to $300 \mathrm{~K}$ over a frequency range between $20 \mathrm{~Hz}$ and $1 \mathrm{MHz}$. The frequency-dependent behavior of the AC conductivity $\sigma_{\mathrm{ac}}(f, T)$ obeys the universal power law, and the values of the effective hopping barrier and hopping distance were found to be $0.79 \mathrm{eV}$ and $6.7 \times 10^{-11} \mathrm{~m}$ from an analysis due to the correlated barrier-hopping model. The dielectric relaxation was identified as a thermally activated non-Debye process involving an activation energy of $41.5 \mathrm{meV}$.
\end{abstract}

Key words: Metal-insulator-semiconductor device, hopping conduction, complex dielectric modulus, relaxation time

\section{INTRODUCTION}

Titania $\left(\mathrm{TiO}_{2}\right)$ has been extensively studied in thin film form for potential applications in oxide electronics such as solar cells, electronic devices, chemical sensors and photocatalysts because of its robust chemical stability under acidic and oxidative environments, wide band gap $(>3 \mathrm{eV})$ and low production cost. ${ }^{1}$ Titania exists in a number of crystalline forms, the most important of which are anatase, rutile and brookite, and the high optoelectronic properties of non-toxic oxides are found to be a phase-dependent quantity. ${ }^{2,3}$ Thin film formulation of the materials can be achieved by using different techniques including DC reactive magnetron sputtering ${ }^{4}$ and electrodeposition. ${ }^{5}$ The magnetron-sputtered $\mathrm{TiO}_{2}$ layers on aluminium alloy 1050 substrates showed columnar growth with increasing crystallite sizes, with an increase in coating thickness leading to an increase in photocatalytic activity. ${ }^{6}$ An electrodeposited ultrathin anatase $\mathrm{TiO}_{2}$ film on fluorine-doped tin oxide has been found to demonstrate a power efficiency of $13.6 \%$ as a blocking layer in perovskite solar cells. ${ }^{7}$

(Received January 5, 2017; accepted June 30, 2017; published online July 31,2017 )
Sol-gel derived $\mathrm{TiO}_{2}$ films exhibit several physically interesting characteristics dependent upon the post-deposition treatment. For example, the photosensitivity of sol-gel derived $\mathrm{TiO}_{2}$ films annealed at $350^{\circ} \mathrm{C}$ was found to be higher than that of its powder by a factor as large as two orders of magnitude. ${ }^{8} \mathrm{TiO}_{2}$ films became anatase in structure when subjected to heat treatment at $600^{\circ} \mathrm{C}$, and the grain sizes were found to be smaller than those found for microwaveexposed films, resulting in the blue shift in UV-visible absorption spectra. ${ }^{9}$ However, the sol-gel synthesis of $\mathrm{TiO}_{2}$ nanoparticles was performed with room temperature ionic liquid 1-n-butyl-3-methylimidazolium hexafluorophosphate as a reaction medium, and the anatase phase was achieved without heat treatment. ${ }^{10}$ The photocatalytic efficiency increases with the increasing concentration of nanostructure $\mathrm{TiO}_{2}$, and the degradation percent can reach $100 \%$ at the optimal catalyst concentration. Anatase $\mathrm{TiO}_{2}$ nanowire arrays were deposited into the nanochannels of an anodic aluminium oxide template by an electrochemically-induced sol-gel method. ${ }^{11} \mathrm{TiO}_{2}$ nanotubes were also prepared using the sol-gel technique for the fabrication of dye-sensitized solar cells, and the high surface area of the nanotubes produced an efficiency of $4 \%$ under $\mathrm{Hg}$-Xe lamp rradiation. ${ }^{12}$ The real part of the dielectric permittivity of sol-gel derived anatase $\mathrm{TiO}_{2}$ cylindrical 
pellets may be tuned by the application of increasing DC bias with a view to lowering barrier heights at the grain boundary. The tunability of $\mathrm{TiO}_{2}$ nanocrystals with grain sizes of $6 \mathrm{~nm}$ is found to be in the order of $\sim 85 \%$ at $100 \mathrm{~Hz}$ and this value is believed to be suitable for phased array antenna applications. ${ }^{13}$ The photocatalytic activity of sol-gel-coated porous tetragonal anatase $\mathrm{TiO}_{2}$ /polystyrene thin films, which were deposited on indium tin oxide substrates using a templating method, showed improved photodegradation of methylene blue under ultraviolet light irradiation compared to porous $\mathrm{TiO}_{2}$ films. This enhancement in photocatalytic activity may be attributed to an increased surface area of $\mathrm{TiO}_{2} /$ polystyrene. ${ }^{14}$ Cystine-modified $\mathrm{TiO}_{2}$ films are found to be more photocatalytically active than $\mathrm{TiO}_{2}$ in splitting water under normal sunlight irradiation. ${ }^{15}$

Metal-insulator-semiconductor (MIS) structures were prepared using solution-processed $191 \mathrm{~nm}$ thick $\mathrm{TiO}_{2}$ films sandwiched between (100) $n$-gallium arsenide (GaAs) substrates and copper $(\mathrm{Cu})$ counter electrodes. These devices exhibited rectifying steady-state conduction behavior with increasing the barrier height from $0.23 \mathrm{eV}$ to $0.63 \mathrm{eV}$ and decreasing the ideality factor from 5.92 to 1.66 in the temperature $T$ range of $50 \mathrm{~K} \leq T \leq 290 \mathrm{~K}^{16}$

We have reported the preparation of non-porous nanostructured anatase titanium dioxide thin films by spin-coating of a spreading solution of titanium isopropoxide, ethanol and acetic acid in the molar ratio of 1:9:0.1 onto a variety of substrates, including microscopic glass slides, silicon and indium tin oxide-coated glass substrates. ${ }^{17,18}$ A trap-controlled space charge-limited mechanism was found to be responsible for charge transport at a high field. Optical absorption was believed to be due to an allowed indirect transition over the optical gap of approximately $3.2 \mathrm{eV} .{ }^{19}$ MIS configurations were fabricated by depositing $37 \mathrm{~nm}$ thick spun $\mathrm{TiO}_{2}$ films on $305 \mu \mathrm{m}$ thick (100) $p$-type silicon (p-Si) substrates. The surface density at the $\mathrm{Si} / \mathrm{TiO}_{2}$ interface and threshold voltage were estimated to be $13.3 \times 10^{14} \mathrm{~m}^{-3}$ and $-85 \mathrm{mV}$, respectively. ${ }^{20}$ The influence of doping has been found to be important because of the change in surface topographies in the MIS structures. A two orders of magnitude increase in rectifying ratio and a simultaneous two-fold decrease of ideality factor have recently been reported for $\mathrm{TiO}_{2}$ films doped with $0.1 \%$ zirconium $1 \%$ by mass in $\mathrm{Al} / \mathrm{TiO}_{2} / \mathrm{p}$-Si MIS structures. No significant change was observed for interface surface density. ${ }^{21}$

A non-destructive alternating current (AC) impedance spectroscopic technique is regarded as being very suitable for identifying the prevalent conduction mechanism, dielectric relaxation processes, and the nature of barrier height and interfacial layer in the MIS structures, when a small AC signal is applied as input perturbation over frequency and temperature ranges. ${ }^{22} \mathrm{AC}$ measurements were performed on $1 \mathrm{~mm}$ thick anatase $\mathrm{TiO}_{2}$ pellets doped in situ with molybdenum nanoparticles $\left(\mathrm{TiO}_{2}-\mathrm{Mo}\right)$ in the frequency $(f)$ range of $40 \mathrm{~Hz} \geq f \leq 100 \mathrm{kHz}$ over $353 \mathrm{~K} \geq T \geq 883 \mathrm{~K}$. Proton conduction along the $\mathrm{TiO}_{2}$-Mo surfaces is believed to be responsible for the increase in conductivity. ${ }^{23} \mathrm{DC}$ magnetronsputtered $30 \mathrm{~nm}$ thick $\mathrm{TiO}_{2}$ films on $p$-GaAs (110) in the MIS structure showed the universal power-law dependence of AC conductivity $\sigma_{\mathrm{ac}}(f, T)$ on $f(1 \mathrm{kHz} \leq f \leq 1 \mathrm{MHz})$ for a temperature range of $80 \mathrm{~K} \geq T \geq 290 \mathrm{~K}$. The results imply the existence of non-random distribution of hopping centers and surface states at the $\mathrm{GaAs} / \mathrm{TiO}_{2}$ interface. ${ }^{24,25} \mathrm{How}$ ever, reports of $\mathrm{AC}$ impedance measurements on MIS structures involving sol-gel $\mathrm{TiO}_{2}$ films are limited. The present article presents the results of $\mathrm{AC}$ electrical measurements to study the dielectric behavior of $\mathrm{TiO}_{2}$ on (100) $p$-type silicon substrates.

\section{EXPERIMENTAL}

By employing a standard photoresist spinner (Microsystem model 4000) at a speed of $5000 \mathrm{rpm}$, a small volume of the spreading solution was spincoated on ultrasonically cleaned (100) $p$-type silicon (p-Si) substrates to form $\mathrm{TiO} 2$ films in the MIS structure shown in Fig. 1 for AC electrical experimental studies. The as-deposited film was then annealed at $550^{\circ} \mathrm{C}$ for a further $30 \mathrm{~min}$ after slowtemperature ramping from room temperature, and then the film was slowly cooled from $550^{\circ} \mathrm{C}$ to room temperature in order to achieve the non-porous anatase structure. The film thickness, $d$, was estimated to be $37.45 \pm 0.15 \mathrm{~nm}$ from the spectroscopic ellipsometeric measurement. The $50 \mathrm{~nm}$ thick aluminium $(\mathrm{Al})$ contacts were thermally evaporated at a rate of $1 \mathrm{nms}^{-1}$ under a vacuum of $10^{-4} \mathrm{~Pa}$ in an Edwards E306A evaporation system in order to complete the MIS structure shown in Fig. 1 for electrical measurements. The active area, $S$, of the device was $7 \times 10^{-6} \mathrm{~m}^{2}$. The details of the preparation of the MIS device structure involving sol-gel derived non-porous $\mathrm{TIO}_{2}$ films are available from our earlier publications. ${ }^{17,20}$ AC measurements were made on the $\mathrm{TiO}_{2}$ film as a function of the frequency, $f$, ranging from $20 \mathrm{~Hz}$ to $10 \mathrm{MHz}$ using a Hewlett Packard 4284 LCR meter and an Oxford Instruments constant bath cryostat in a microprocessor-controlled measuring system over a temperature $(T)$ range of $100-360 \mathrm{~K}$. During the entire measurements, the temperature stability was maintained in the order of $\pm 0.5 \mathrm{~K}$. The direct current (DC) bias was maintained at zero volts during the measurement to allow the device to be operated in an accumulation regime.

\section{RESULTS AND DISCUSSION}

Experimental results are presented along with the interpretation in order to identify the mechanisms responsible for charge transport and dielectric relaxation in $\mathrm{Al} / \mathrm{TiO}_{2} / \mathrm{p}-\mathrm{Si} /(100)$ MIS structures. New information has been elucidated from a careful 


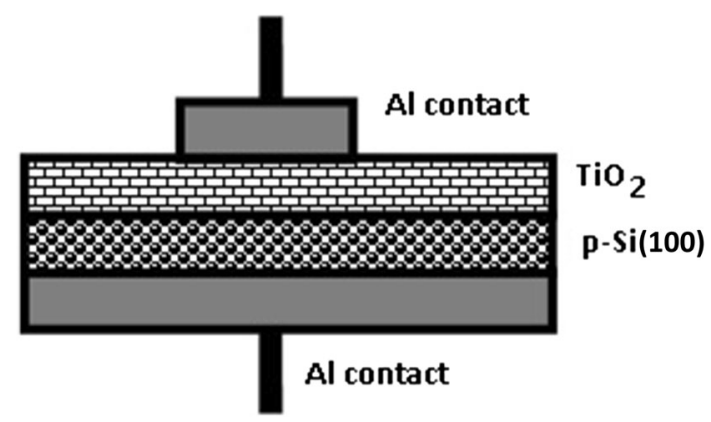

Fig. 1. A schematic diagram of the metal-insulator-semiconductor (MIS) structure containing $37.45 \mathrm{~nm}$ thick sol-gel derived $\mathrm{TiO}_{2}$ film on $305 \mu \mathrm{m}$ thick (100) $p$-type silicon substrates sandwiched between two $50 \mathrm{~nm}$ thick aluminium (Al) contacts $\left(\mathrm{Al} / \mathrm{p}-\mathrm{Si} / \mathrm{TiO}_{2} / \mathrm{Al}\right)$.

comparison of the values of physical parameters estimated in this investigation with published data.

\section{Dependence of Conductivity on Frequency and Temperature}

Figure 2 presents a set of reproducible curves on a double logarithmic scale showing the frequency dependence of $\mathrm{AC}$ conductivity, $\sigma_{\mathrm{ac}}(f, T)$, of spun $\mathrm{TiO}_{2}$ thin films at eight different temperatures, $T$, between $100 \mathrm{~K}$ and $360 \mathrm{~K}$. The temperature dependence of $\sigma_{\mathrm{ac}}(f, T)$ became more pronounced for the lowfrequency compared to the high-frequency regime with a transition frequency of $100 \mathrm{kHz}$. Three distinct regimes, characteristic of particular temperature and frequency ranges, are found to exist for conduction behavior. Firstly, a plateau region of frequency conductivity $\sigma_{\mathrm{T}}(0)$ at a temperature $T$ was observed over the low-frequency range $f \leq 100 \mathrm{kHz}$ for $T \geq 300 \mathrm{~K}$. The existence of a similar region $\sigma_{\mathrm{T}}(0)$ was observed in the $\sigma_{\mathrm{ac}}(f, T)$ curves for the chemical vapur-deposited rutile $\mathrm{TiO}_{2}$ films on silicon substrates at a temperature of $562 \mathrm{~K} \geq T \leq 870 \mathrm{~K}$ and a frequency of $100 \mathrm{~Hz} \geq f \leq 10^{4} \mathrm{~Hz}^{26}$ This value is believed to be contributing to the DC conductivity, and this DC contribution becomes so dominant at $T \geq 1000 \mathrm{~K}$ that $\sigma_{\mathrm{ac}}(f, T)=\sigma_{\mathrm{T}}(0)$ over $0.1 \mathrm{~Hz} \geq f \leq 10^{6} \mathrm{~Hz}^{27}$ The second regime is related to an increase in $\sigma_{\mathrm{ac}}(f, T)$ from $\sigma_{\mathrm{T}}(0)$ at a cross-over frequency of $f \approx 10 \mathrm{kHz}$. Thirdly, $\sigma_{\mathrm{ac}}(f, T)$ is found to be increasingly independent of temperature for the high- frequency range $f \geq 100 \mathrm{kHz}$. The frequency dependence of conductivity $\sigma_{\mathrm{ac}}(f, T)$, for amorphous semiconductors and disordered systems is generally expressed in a form of the universal power law relationship ${ }^{28}$ :

$$
\sigma_{\mathrm{ac}}(f, T)=\sigma_{\mathrm{T}}(0)+A f^{s}
$$

where $A$ is a constant which is dependent on temperature. The value of the frequency exponent, $s$, usually lies between the values of zero and unity, and the conduction mechanism can be identified from the temperature-dependent behavior of $s$. Values of the exponent $s$ as a function of

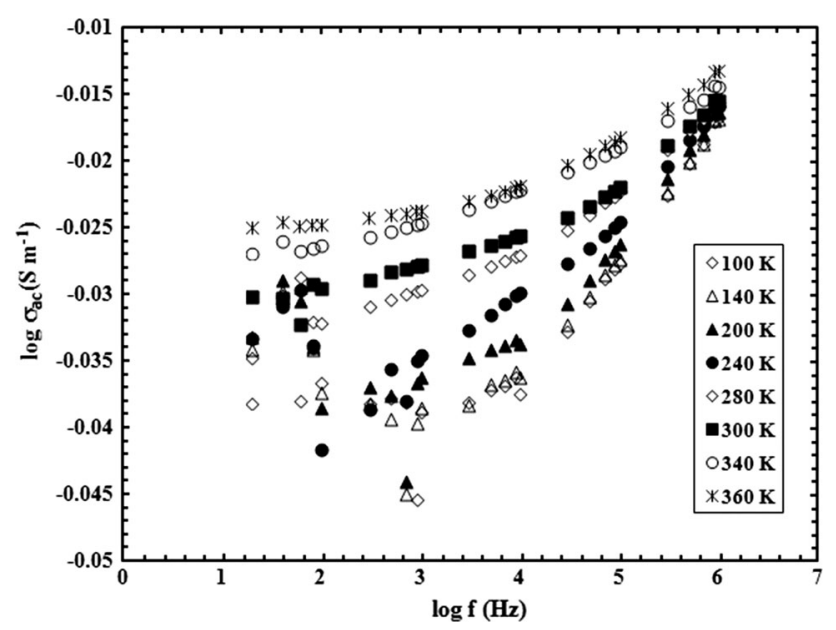

Fig. 2. Frequency dependence of $\mathrm{AC}$ conductivity $\sigma_{a c}(f, T)$ for nonporous anatase $\mathrm{TiO}_{2}$ in the $\mathrm{Al} / \mathrm{p}-\mathrm{Si} / \mathrm{TiO}_{2} / \mathrm{Al}$ MIS structure at temperatures increasing from $100 \mathrm{~K}$ to $360 \mathrm{~K}$, measured with the stability of $\pm 0.5 \mathrm{~K}$.

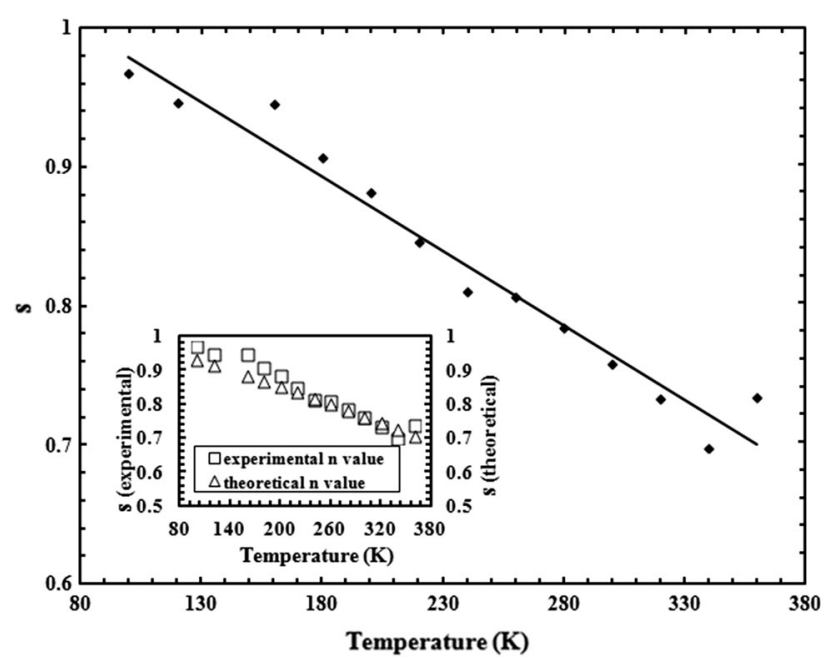

Fig. 3. Frequency exponent $s$ as a function of temperature for the $\mathrm{TiO}_{2}$ sample and, inset, the best fitted parameters of the values of the effective-hopping barrier $W_{M}$ and characteristic relaxation time $\tau_{0}$.

temperature were calculated from the slope of $\log \sigma_{\mathrm{ac}}-\sigma_{\mathrm{T}}(0)$ against a $\log f$ plot, and these values were found to lie in the range of $0.7 \geq s \leq 0.9$ shown in Fig. 3. The exponent $s$ decreases smoothly with increasing $T$, and is independent of frequency in the investigated frequency range. The frequencyindependent conductivity $\sigma_{\mathrm{T}}(0)$ is obtained by extrapolation of the conductivity as it approaches the frequency of zero.

Different theoretical models such as quantum mechanical tunnelling (QMT), classical hopping over barrier (HOB) and correlated barrier hopping $(\mathrm{CBH})$ have been proposed for explaining AC conduction in disordered thin films. The QMT model involving electrons as carriers predicts a 
temperature-independent value for $s$. Small polarons are not believed to be responsible for the AC conduction in $\mathrm{TiO}_{2}$, since the increase of $s$ with the rise of temperature $T$ is expected according to this model. According to large polaron QMT models, $s$ decreases to a minimum at a certain temperature and subsequently increases with $T$, and this is not a valid conduction mechanism in the present investigation. The HOB model predicts a constant value of unity for $s$ since the barrier heights are not dependent on the intersite separation. ${ }^{29}$ In the CBH models, bipolar hopping takes place involving two electrons or holes over the barrier height $W_{\mathrm{M}}$ separated by a hopping distance $R_{\omega}$. In this model, the exponent $n$ increases to unity as $T \rightarrow 0 \mathrm{~K}^{30}$ The observed dependence of $s$ on $T$ for the $\mathrm{TiO}_{2}$ film under the present investigation is consistent with the prediction of the $\mathrm{CBH}$ model. $\mathrm{CBH}$ conduction has recently been reported for rutile $\mathrm{TiO}_{2}$ thin films, deposited by $\mathrm{RF}$ magnetron sputtering using a powder target, exhibiting the decrease of $s$ from 0.8 to 0.4 with the increment in $T$ within the range of $660 \mathrm{~K}<T<740 \mathrm{~K}^{31}$

The dependence of the frequency exponent, $s$, on temperature $T$ is written in the form ${ }^{32}$

$$
s=1-\frac{6 k_{\mathrm{B}} T}{W_{\mathrm{M}}-k_{\mathrm{B}} T \ln \left(\frac{1}{2 \pi f \tau_{\mathrm{c}}}\right)}
$$

where $k_{\mathrm{B}}=8.62 \times 10^{-5} \mathrm{eV} \mathrm{k}^{-1}$ is the Boltzmann constant, and $W_{\mathrm{M}}$ and $\tau_{\mathrm{c}}$ are the effective hopping barrier and the characteristic relaxation time, respectively. The inset in Fig. 3 shows the experimental and fitting data from Eq. 2 as a function of temperature, and good agreement exists between the theoretical and experimental values of $s$. The values of $0.79 \mathrm{eV}$ and $1 \times 10^{-8} \mathrm{~s}$ are obtained for $W_{\mathrm{M}}$ and $\tau_{0}$ from the best fitted parameters to Eq. 2.

The hopping distance $R_{\mathrm{f}}$ at a particular frequency and temperature respectively are given in the form $^{33}$ :

$$
R_{\mathrm{f}}=\frac{n_{\mathrm{el}} e^{2}}{\pi \varepsilon_{0} \varepsilon_{\mathrm{ox}}\left[W_{\mathrm{M}}+k_{\mathrm{B}} T \ln \left(2 \pi f \tau_{\mathrm{c}}\right)\right]}
$$

where $n_{\mathrm{el}}$ is the number of electrons involved in the process of hopping between centers and $n_{\mathrm{el}}=1$ and $n_{\mathrm{el}}=2$ for and $n_{\mathrm{el}}=2$ for single polaron and bipolaron hopping, respectively. The quantities $\varepsilon_{0}=8.85 \times 10^{-12} \mathrm{Fm}^{-1}$ and $\varepsilon_{\mathrm{ox}}=13$ are the dielectric permittivity of the free space and the $\mathrm{TiO}_{2}$ film, ${ }^{20}$ respectively. With the knowledge of $W_{\mathrm{M}}$ and $\tau_{\mathrm{c}}$, the hopping distance $R_{\mathrm{f}}$ is found from Eq. 3 to be $6.7 \times 10^{-11} \mathrm{~m}$.

\section{Dielectric Relaxation}

The variation in capacitance $C$ with frequency within the range of $20 \mathrm{~Hz} \geq f \leq 10^{6} \mathrm{~Hz}$ at various temperatures for the same MIS structure is shown in Fig. 4. The capacitance is found to be nearly

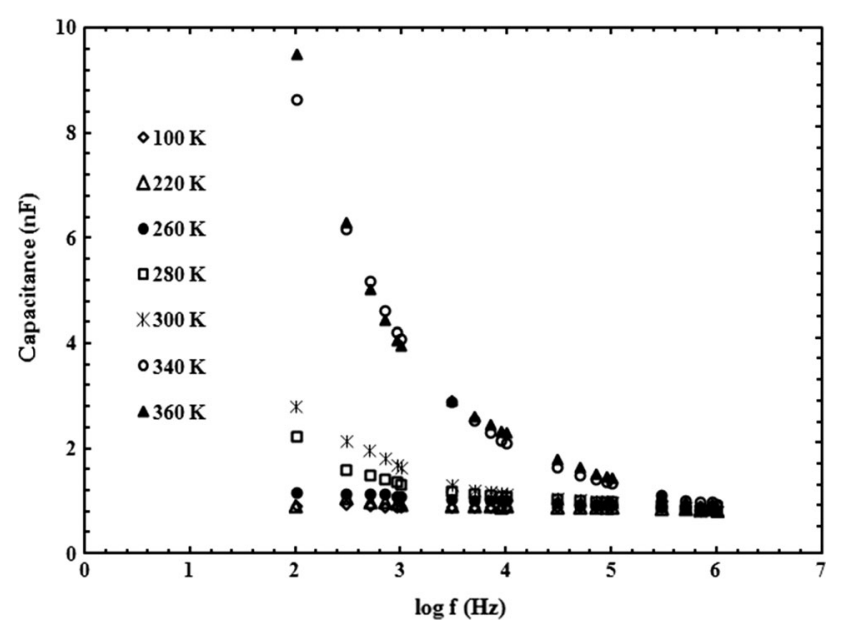

Fig. 4. Frequency dependence of $\mathrm{AC}$ capacitance $\mathrm{C}$ for $\mathrm{TiO}_{2}$ the $\mathrm{Al} /$ $\mathrm{p}-\mathrm{Si} / \mathrm{TiO}_{2} / \mathrm{Al} \mathrm{MIS}$ structure at seven temperatures ranging from $100 \mathrm{~K}$ to $360 \mathrm{~K}$ measured with a stability of $\pm 0.5 \mathrm{~K}$.

independent of frequency within the temperature range of $T \geq 300 \mathrm{~K}$, whereas the capacitance decreases rapidly with increasing frequency for $T>300 \mathrm{~K}$. The capacitance subsequently approaches the low-temperature value at high frequencies.

The experimental data were analyzed in terms of the real $M^{\prime}$ and imaginary $M^{\prime \prime}$ parts of the complex dielectric modulus. $M^{\prime}$ and $M^{\prime \prime}$ can be defined in terms of the real $\left(\varepsilon^{\prime}\right)$ and imaginary $\left(\varepsilon^{\prime \prime}\right)$ parts of the complex dielectric constant in the following forms ${ }^{34}$ :

$$
\begin{gathered}
M^{\prime}=\frac{\varepsilon^{\prime}}{\left(\varepsilon^{\prime \prime 2}+\varepsilon^{\prime \prime 2}\right)} \\
M^{\prime \prime}=\frac{\varepsilon^{\prime \prime}}{\left(\varepsilon^{\prime 2}+\varepsilon^{\prime \prime 2}\right)} \\
\text { where } \varepsilon^{\prime}=\frac{C d}{S \varepsilon_{0}} \text { and } \varepsilon^{\prime \prime}=\frac{\sigma_{\mathrm{ac}}}{2 \pi f \varepsilon_{0}}
\end{gathered}
$$

The modulus formalism is regarded as being a useful tool for the interpretation of impedance data over the ranges of frequency and temperature in respect of electrode polarization, grain boundary conduction effect, bulk properties and electrical conductivity. ${ }^{35}$ Figure $5 \mathrm{a}$ and $\mathrm{b}$ shows the frequency dispersion of the real and imaginary parts of the dielectric modulus for spun $\mathrm{TiO}_{2}$ films at selected temperatures. The real part $M^{\prime}$ in Fig. $5 \mathrm{a}$ is found to decrease with the rise in temperature. A nearly temperature-independent maximum asymptotic value was observed at the high frequency, $f \geq 10^{5} \mathrm{~Hz}$, while at a low frequency, $f \leq 10^{3} \mathrm{~Hz}$, high temperature values of $M^{\prime}$ tend to zero faster than those corresponding to low temperatures, $T \leq 280 \mathrm{~K}$. As shown in Fig. 5b, the frequency dispersion of the imaginary part $M^{\prime \prime}$ of the dielectric modulus exhibits broad asymmetrical peaks, 
(a)

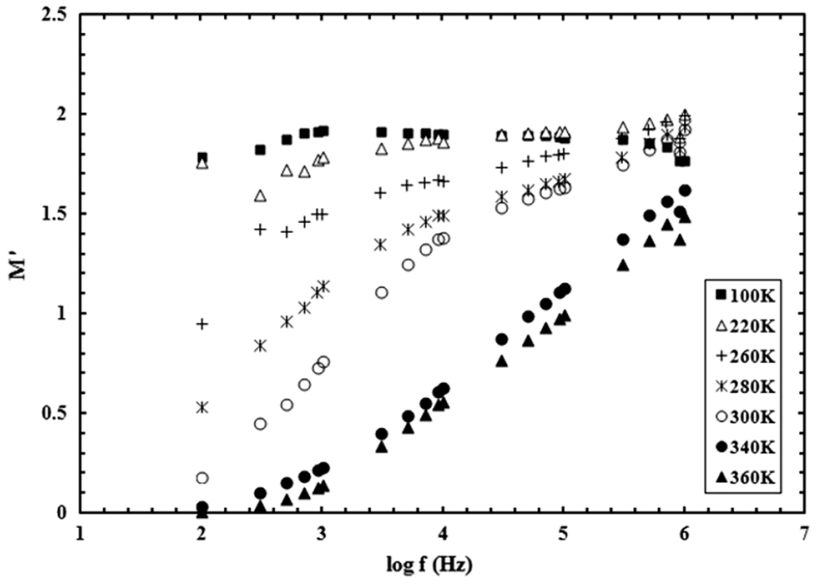

(b)

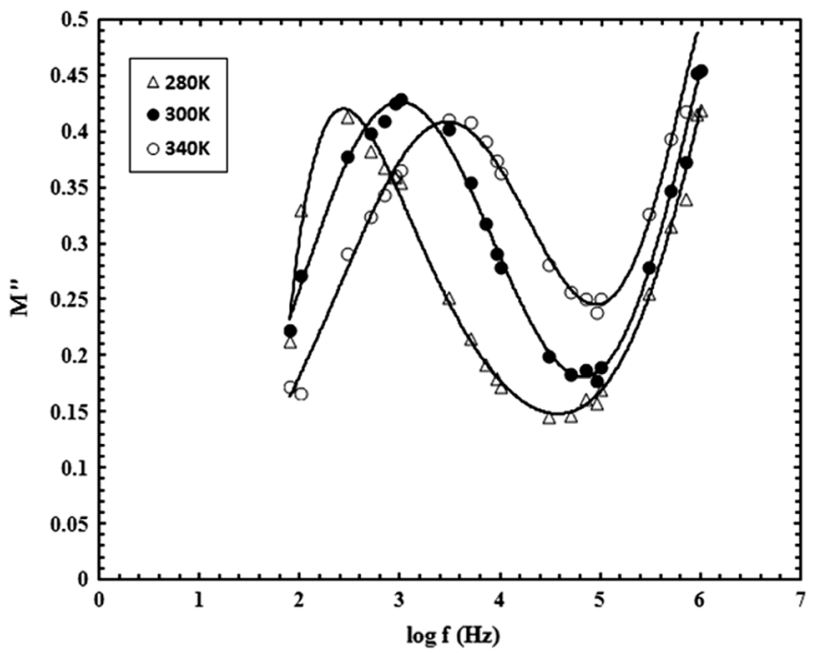

(c)

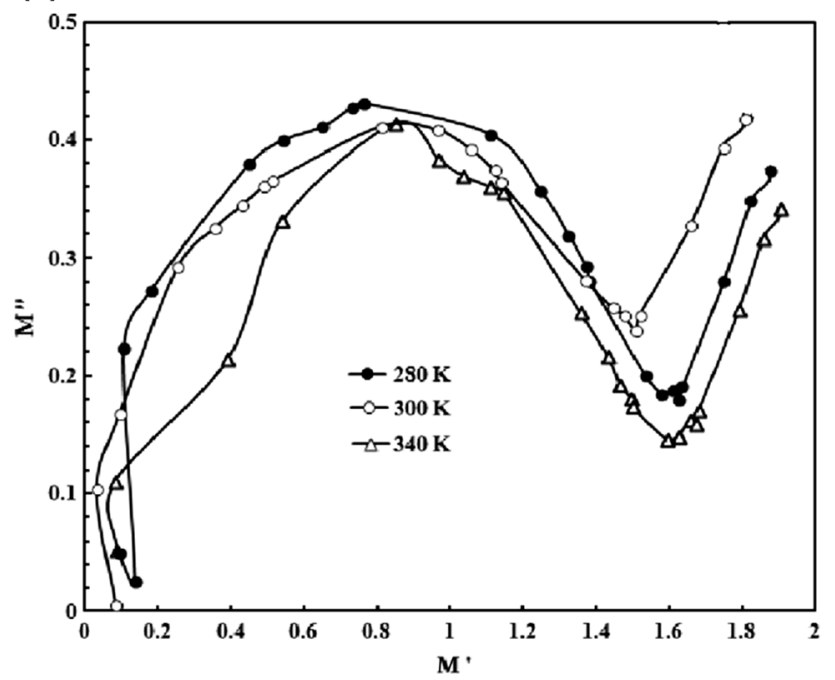

Fig. 5. (a) Real part $M^{\prime}$ and (b) imaginary part $M^{\prime}$ of the dielectric modulus $M$ as a function of frequency at specified temperatures increasing from $100 \mathrm{~K}$ to $360 \mathrm{~K}$. (c) Cole-Cole plots of the imaginary part $M^{\prime \prime}$ versus the real part $M^{\prime \prime}$ at the selected temperatures.

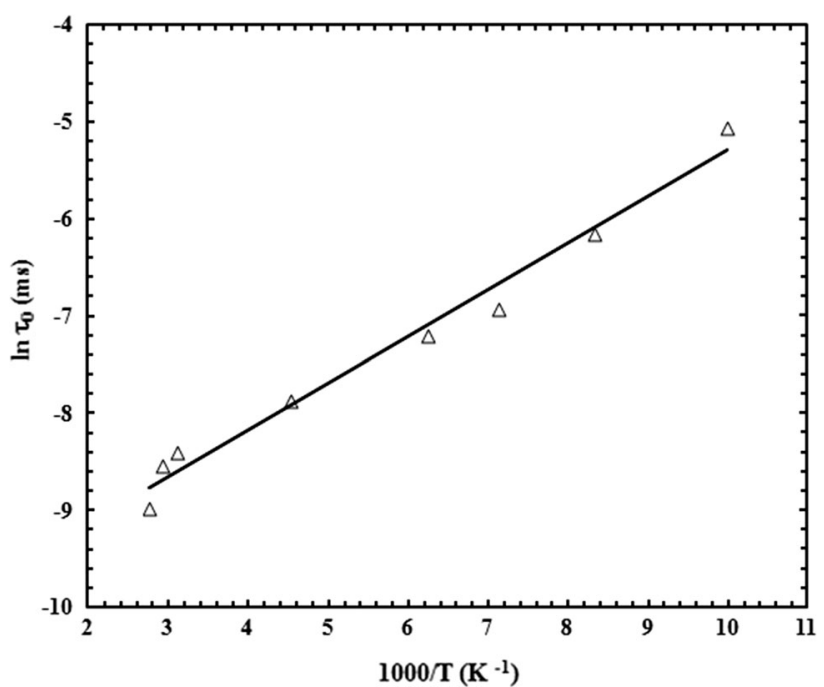

Fig. 6. Arrhenius plot of $\ln \tau_{0}$ as a function of the inverse of temperature.

systematically shifting towards higher frequencies with the increase of temperature. This implies that the relaxation process is thermally activated and that the charge mobility increases with the rise in temperature, leading to the reduction in dielectric relaxation. The broad nature of the peak represents the distribution of relaxation times $\tau=1 / 12 \pi f$, and the center of the relaxation peak in Fig. $5 \mathrm{~b}$ is usually identified by the most probable frequency, $f_{0}$. Charge carriers are mobile over long distances for $f<f_{0}$, while the charge carriers, being confined within potential wells, are mobile over a short-range potential $f>f_{0}$. The Debye model is related to an ideal frequency response of localized relaxation, and the absence of overlapping peaks in Fig. $5 \mathrm{~b}$ indicates that the relaxation process is of a non-Debye type. $^{36}$ This frequency dispersive behavior is generally described in terms of the non-linear Kohlrausch-Williams-Watts decay function and the stretched constant $\beta$, which can in principle be estimated from the full width of the bell-shaped curves in Fig. 5b at half maximum. ${ }^{37}$ The values of the stretched constant $\beta$ are found to increase from 0.4359 to 0.8217 as the temperature $T$ is decreased from $320 \mathrm{~K}$ to $280 \mathrm{~K}$, and $\beta \neq 1$ is also consistent with non-Debye-type relaxation. Nearly semicircular behavior is observed in the Cole-Cole plot in Fig. 5c of $M^{\prime \prime}$ against $M^{\prime}$ for these temperatures, possibly due to the decrease of modulus resistance. The decreasing temperature produced the relatively small shift of $M^{\prime \prime}$ to higher values, and this observation may be interpreted in terms of a gradually increasing grain boundary contribution to conduction. ${ }^{24}$

For further investigation into the effect of temperature on relaxation, the relaxation time $\tau_{0}$ 
$\left(\tau_{0}=1 / 12 \pi f_{0}\right)$ at the different temperatures was determined from the reciprocal of the peak frequency. The Arrhenius plot of $\ln \tau_{0}$ as a function of the inverse of temperature is shown in Fig. 6, and the relaxation is believed to be due to a thermally activated process given in the form:

$$
\tau_{0}=A \exp \left(\frac{E_{\mathrm{a}}}{k_{\mathrm{B}} T}\right)
$$

where $A$ is the pre-exponential factor.

The decrease in dielectric relaxation time with the rise of temperature occurs due to the increasing charge mobility at high temperature. The value of the activation energy of dielectric relaxation, $E_{\mathrm{a}}$, is found to be $41.5 \pm 0.99 \mathrm{meV}$. The dielectric relaxation behavior of electrospun $\mathrm{TiO}_{2}$ nanofibers between two $\mathrm{Al}$ electrodes on glass substrates has been investigated for the frequency range of $1 \mathrm{~Hz} \geq f \geq 1 \mathrm{MHz}$ at selected temperatures over $333 \mathrm{~K} \geq T \geq 513 \mathrm{~K}$, and the resulting frequency dispersion of the dielectric moduli shows the characteristic features similar to those observed in the present investigation. However, the value of $E_{\mathrm{a}}$ is smaller than that obtained for $\mathrm{TiO}_{2}$ nanofibers by nearly two orders of magnitude, and the difference may be attributed to the quantum size effect in the nanofibers. $^{38}$

\section{CONCLUSION}

Nanostructured sol-gel derived anatase titania films $37 \mathrm{~nm}$ thick were deposited at room temperature on ultrasonically cleaned (100) $p$-silicon substrates to fabricate metal-insulator-semiconductor (MIS) structures using $50 \mathrm{~nm}$ thick thermal aluminium contacts. AC impedance measurements were performed on MIS structures at different temperatures ranging from $100 \mathrm{~K}$ to $300 \mathrm{~K}$ over a frequency range of $20 \mathrm{~Hz}$ to $1 \mathrm{MHz}$. The dependence of AC conductivity on frequency was found to support the universal power law, with the exponent decreasing with the rising temperature. This behavior of the power law exponent was analyzed in terms of the correlated barrier-hopping model. The carrier transport was believed to be thermally-assisted hopping over the potential barrier of $0.79 \mathrm{eV}$ across a distance of $6.7 \times 10^{-11} \mathrm{~m}$ between defect centers. The dielectric relaxation was interpreted using the modulus formalism, and the frequency corresponding to the peak of frequency dispersion of the imaginary part of the dielectric moduli was associated with the dielectric relaxation time, and a value of $41.5 \mathrm{meV}$ was obtained for the energy required for a thermally-activated dielectric relaxation process.

\section{ACKNOWLEDGEMENT}

Dr. R. Capan is grateful to the Leverhulme Trust Foundation for the award of a fellowship to visit the UK. Gratitude is also due to Dr. Lesley Hanna, Dr. Myles Worsley and Miss Virginia Martin Torrejon of Institute of Materials and Manufacturing, Brunel University London, for help in preparing the manuscript.

\section{OPEN ACCESS}

This article is distributed under the terms of the Creative Commons Attribution 4.0 International License (http://creativecommons.org/licenses/by/4.0/), which permits unrestricted use, distribution, and reproduction in any medium, provided you give appropriate credit to the original author(s) and the source, provide a link to the Creative Commons license, and indicate if changes were made.

\section{REFERENCES}

1. D. Koziej, A. Alessandro, and M. Niederberger, Adv. Mater. 26, 235 (2014).

2. K.K. Saini, S.D. Sharma, M. Chanderkant, D.Singh Kar, and C.P. Sharma, J. Non-Cryst. Solids 353, 2469 (2007).

3. J.Y. Kim, H.S. Jung, J.H. No, J.R. Kim, and K.S. Hong, J. Electroceram. 16, 447 (2006).

4. A. Vale, N. Chaure, M. Simonds, A.K. Ray, and N. Bricklebank, J. Mater. Sci. Mater. Electron. 17, 851 (2006).

5. C.F.C. Blanco, C. Pal, J.J. Ojeda, A.K. Ray, and A.K. Sharma, J. Electrochem. Soc. 159, E30 (2012). doi: 10.1149/2.016202jes.

6. S. Daviosdottir, R. Shabadi, A.C. Galca, I.H.I. Andersen, K. Dirscherl, and R. Ambat, Appl. Surf. Sci. 313, 677 (2014). doi:10.1016/j.apsusc.2014.06.047.

7. T.S. Su, T.Y. Hsieh, C.Y. Hong, and T.C. Wei, Sci. Rep. 5, 16098 (2015). doi:10.1038/srep16098.

8. A.E.J. Gonzalez and S.G. Santiago, Sem. Sci. Technol. 22, 709 (2007).

9. D.D. Claudio, A.R. Phani, and S. Santucci, Opt. Mater. 30, 279 (2007).

10. Y.G. Zhai, Y. Gao, F.Q. Liu, Q. Zhang, and G. Gao, Mater. Lett. 61, 5056 (2007).

11. L. Sun, J. Zuo, Y.K. Lai, C.G. Nie, and C.J. Lin, Acta Phys. Chim. Sin. 23, 1603 (2007).

12. I.C. Flores, J.N. de Freitas, C. Longo, M.A. de Paoli, H. Winnischofer, and A.F. Nogueira, J. Photochem. Photobiol. A Chem. 189, 153 (2007).

13. T. Prakash, A.T. Selvan, and S.N.S. Begum, Superlattices Microstruct. 91, 182 (2016).

14. A. Bourezgui, I. Kacem, I. Ben Assaker, M. Gannouni, J. Ben Naceur, M. Karyaoui, and R. Chtourou, J. Porous Mater. 23, 1085 (2016). doi:10.1007/s10934-016-0166-3.

15. X. Liu, L.J. Chen, R.Y. Chen, Z. Chen, X. Chen, and X. Zheng, Res. Chem. Intermed. 41, 3623 (2015). doi: 10.1007/s11164-013-1476-6.

16. S. Sonmezoglu and S. Akin, Curr. Appl. Phys. 12, 1372 (2012). doi:10.1016/j.cap.2012.03.030.

17. Q. Fan, B. McQuillin, A.K. Ray, M.L. Turner, and A.B. Seddon, J. Phys. D 33, 2683 (2000).

18. A.K. Hassan, N.B. Chaure, A.K. Ray, A.V. Nabok, and S. Habesch, J. Phys. D 36, 1120 (2003).

19. R. Capan, N.B. Chaure, A.K. Hassan, and A.K. Ray, Sem. Sci. Technol. 19, 198 (2004).

20. N.B. Chaure, A.K. Ray, and R. Capan, Sem. Sci. Technol. 20, 788 (2005).

21. I.H. Tasdemir, O. Vural, and I. Dokme, Philos. Mag. 96, 1684 (2016). doi:10.1080/14786435.2016.1178403.

22. J.R. Macdonald, Impedance Spectroscopy-Emphasizing Solid Materials and Systems (New York: Wiley, 1987).

23. R. Bargougui, N. Bouazizi, S. Ammar, and A. Azzouz, J. Electron. Mater. 46, 85 (2017). doi:10.1007/s11664-016-4947-x.

24. Y.S. Asar, T. Asar, S. Altindal, and S. Ozcelik, Philos. Mag. 95, 2885 (2015). doi:10.1080/14786435.2015.1081301.

25. Y.S. Asar, T. Asar, S. Altindal, and S. Ozcelik, J. Alloy. Compd. 628, 442 (2015). doi:10.1016/j.jallcom.2014.12.170.

26. D.Y. Guo, A. Ito, T. Goto, R. Tu, C.B. Wang, Q. Shen, and L.M. Zhang, J. Mater. Sci. Mater. Electron. 24, 1758 (2013). 
27. D. Regonini, V. Adamaki, C.R. Bowen, S.R. Pennock, J. Taylor, and A.C.E. Dent, Solid State Ion. 229, 38 (2012).

28. A.K. Jonscher, Nature 267, 673 (1977).

29. A.R. Long, Adv. Phys. 31, 553 (1982).

30. S.R. Elliott, Adv. Phys. 36, 135 (1987).

31. I. Ben Jemaa, F. Chaabouni, and M. Abaab, Phys. Status Solidi A Appl. Mater. 214, 1600426 (2017).

32. N.F. Mott and E. Davis, Electronic Process in Non Crystalline Materials, 2nd ed. (Oxford: Clarendon, 1979).
33. S.R. Elliott, Philos. Mag. 37, 553 (1978).

34. S.R. Elliott, J Non-Cryst. Solids 170, 97 (1994).

35. V.C.V. Gowda, B.K. Chethana, and C.N. Reddy, Mater. Sci. Eng. B 178, 82 (2013).

36. R. Gerhardt, J. Phys. Chem. Solids 55, 1491 (1994).

37. C.K.K. Reddy, G. Suman, R.B. Rao, N.K. Katari, and M.R.P. Reddy, Appl. Nanosci. 6, 1043 (2016).

38. S.S. Batool, Z. Imran, M.A. Rafiq, M.M. Hasan, and M. Willander, Ceram. Int. 39, 1775 (2013). 